\title{
The Association of Plasma Fractalkine and Inflammation After Ischemic Stroke
}

\author{
Lucia Herminawati ${ }^{1,2,}$, Andi Wijaya ${ }^{3,4}$ Mansyur Arief ${ }^{1}$, Suryani As'ad $^{1}$ \\ ${ }^{1}$ Postgraduate Program in Medical Science, Faculty of Medicine, Hasanuddin University, Jl. Perintis Kemerdekaan Km.10, Makassar, Indonesia \\ ${ }^{2}$ Prodia Diagnostic Line, Kawasan Industri Jababeka III Jl. Tekno 1 Blok C2 Unit D-F, Cikarang, Indonesia \\ ${ }^{3}$ Postgraduate Program in Clinical Biochemistry, Faculty of Medicine, Hasanuddin University, Jl. Perintis Kemerdekaan Km.10, Makassar, Indonesia \\ ${ }^{3}$ Prodia Clinical Laboratory, Jl. Cisangkuy No.2, Bandung, Indonesia \\ *Corresponding author. E-mail: lucia.herminawati@proline.co.id
}

Received date: Apr 20, 2016; Revised date: May 13, 2016; Accepted date: May 26, 2016

\section{Abstract}

$\mathrm{B}$ ACKGROUND: Inflammation affects brain after stroke with main functions to rapidly eliminate the source of the disturbance, remove damaged tissue and then restore tissue homeostasis. High sensitive C-reactive protein (hsCRP) is a sensitive marker of inflammation and tissue injury in the arterial wall, while fractalkine is a distinct chemokine that promotes inflammatory signaling after neuronal death on ischemic stroke. We aim to investigate the association of fractalkine with hsCRP as a marker of inflammation in ischemic stroke patients.

METHODS: This study was designed as a cross-sectional study. Soon after patients with ischemic stroke admitted to hospital, plasma fractalkine and hsCRP concentrations were assesed. Subjects had to be at least 30 years old and maximum 30 days of stroke onset. High inflammation was defined as hsCRP value $>3 \mathrm{mg} / \mathrm{L}$.
RESULTS: High fractalkine levels were found on 24 ischemic stroke patients (49\%) and mean of fractalkine 0.719 $\mathrm{ng} / \mathrm{mL}$ on patients with stroke onset $<7$ days was higher than patients with stroke onset 7-30 days. Low fractalkine levels $(<0.527 \mathrm{ng} / \mathrm{mL})$ were found on ischemic stroke patients with onset 7-30 days accompanied by high inflammation (hsCRP $>3 \mathrm{mg} / \mathrm{L}$ ), but no significant correlation between fractalkine and hsCRP $(p=0.613)$.

CONCLUSION: High inflammation and low plasma fractalkine profile was found after 7 days of onset in ischemic stroke patients. No significant correlation between fractalkine and hsCRP in ischemic stroke patients.

KEYWORDS: CRP, fractalkine, inflammation, ischemic stroke

Indones Biomed J. 2016; 8(2): 109-14

\section{Introduction}

Stroke is one of the non-communicable diseases (NCD), a group of diseases causing the death, leading of second ranked in the world after coronary heart disease. It was estimated that about 16 million people affected by the first stroke each year and 5.7 million people died of stroke.(1) Among all types of stroke, ischemic stroke has been found to take place $85 \%$ of stroke incidence.

Inflammation involved in ischemia cascade has important role in the course of stroke.(2) After ischemic stroke, inflammatory process affected the brain and immune cells such as neutrophils and macrophages. Inflammatory process started from the intravascular compartment immediately after the occlusion, followed by hypoxia, increase of shear stress and production of reactive oxygen species (ROS), thereby activating the coagulation cascade that led to activation of complement, platelet, and endothelial cells. Usually referred as post-ischemic inflammation, this process is characterized by a sequence of events involving brain, blood vessels, circulating blood and lymphoid organs.(3) Meanwhile, in the perivascular space, microglia and perifer macrophages, then released the 
peripheral mediators of pro-inflammatory that contributed to the blood-brain barrier damage, including interleukin (IL)-6, which stimulate the synthesis of C-reactive protein (CRP). $(3,4)$

High sensitive CRP (hsCRP) is an acute phase protein which sensitively increase on inflammatory process and tissue injury in the arterial wall. $(5,6)$ Healthy person normally has a median CRP concentration of approximately $1 \mathrm{mg} / \mathrm{L}$, while during tissue injury, inflammation or infection it tends to undergo 100-fold or more increase. Elevated plasma CRP levels have been demonstrated to predict the risk of future ischemic stroke and transient ischemic attack (TIA) in the elderly. Studies have shown a very early increase (within 3 hours) in patients with acute stroke with or without infection, and are an independent predictor of survival or non-fatal vascular event after ischemic stroke.(7)

Chemokines are a family of cytokines or signaling proteins secreted by cells that form a chemical gradient which guides migration of inflammatory cells to the site of injury. Fractalkine is a structurally distinct chemokine, the only chemokine with the CX3C motif (CX3CL1), exists in both a membrane bound and soluble form. In its membrane bound form, CX3CL1 serves as an adhesion molecule for leukocytes, in its soluble form, it serves as a potent chemoattractant for them. $(8,9,10)$

Fractalkine is expressed constitutively in neurons and suppressed microglial activation through its microglial chemokine with the $\mathrm{CX} 3 \mathrm{C}$ motif receptor 1 (CX3CR1) which expressed by monocytes, natural killer cells, T-lymphocytes, macrophages and microglia. After neuronal death, loss of cell-to-cell interaction between neurons and microglia, which are kept quiescent in the normal state by contact with neurons, also promotes inflammatory signaling.(11) Recently, fractalkine has become notable in stroke research, primarily in animal models and in in vitro studies. The roles of fractalkine in ischemic stroke patients is not yet sufficiently investigated and whether fractalkine adopts neuroprotective or neurotoxic functions remains controversial. We aim to investigate the association of fractalkine with hsCRP as a marker of inflammation in ischemic stroke patients.

\section{Methods}

\section{Research Subjects}

This study was designed as cross sectional study of ischemic stroke patients in The Gatot Subroto Army Central Hospital Jakarta, from February 2015 through September 2015.
Subjects had to be at least 30 years old and maximum 30 days of onset stroke. Ischemic stroke was defined according to the American Heart Association/American Stroke Association (AHA/ASA) as an episode of neurological dysfunction caused by focal cerebral, spinal, or retinal infarction. Cerebral infarction was diagnosed on the basis of history, neurological examination, and brain magnetic resonance imaging (MRI). All subtypes of ischemic stroke were included. Subjects with ongoing therapy for malignancy, known history of brain tumor, and those taking immunomodulatory drugs were excluded. Blood was drawn as soon as possible when patients admitted to hospital. The study was approved by ethics committee of Faculty of Medicine, Hasanuddin University, Makassar, Indonesia (approval number: UH14110581).

\section{Subjects Characteristics and Biomarker Assessment}

Baseline clinical information was extracted from the medical records. Subjects' demographic and cardiovascular risk factors such as hypertension, diabetes mellitus, and lipid dysregulation were obtained. Serum hsCRP level was estimated by Immulite chemistry system by Prodia Clinical Laboratory using chemiluminescent immunometric methods. High sensitivity CRP levels was universally standardised and accept a value above $3 \mathrm{mg} / \mathrm{L}$ as high inflammation.(12) Fractalkine levels was determined in ethylenediaminetetraacetic acid (EDTA) plasma using enzyme-linked immunosorbent assay (ELISA) kits (R\&D Systems, Inc., Minneapolis, MN, USA). The sensitivity of fractalkine assay was $0.018 \mathrm{ng} / \mathrm{mL}$.

\section{Statistical Analysis}

Statistical analysis was performed using the Statistical Package for Social Sciences (SPSS 16.0, SPSS Production Facility, Chicago, Illinois, USA). Normality of data distribution was assessed using Shapiro-Wilk test. The non-parametric Spearman rank correlation test was used to investigate the relationship between concentrations of fractalkine and hsCRP. In all statistical analyses, two-sided $p$ values of 0.05 were considered significant.

\section{Results}

In total, 49 patients were recruited voluntarily in our study during eight months period. The age mean of total subjects was 57 years (range, 38-76 years). For further clinical data and risk factor see Table 1. Normality test using ShapiroWilk showed that hsCRP and fractalkine didn't have a 
normal distribution $(p<0.05)$. Based on $3 \mathrm{mg} / \mathrm{L}$ as the cut off point for hsCRP, we found 18 patients (36.7\%) with low inflammation (hsCRP $<3 \mathrm{mg} / \mathrm{L}$ ) and 31 patients (63.3\%) with high inflammation (hsCRP $\geq 3 \mathrm{mg} / \mathrm{L}$ ) in total subjects. Fractalkine median levels of total subjects was found 0.527 $\mathrm{ng} / \mathrm{mL}$. Based on this median value, we divided subjects into low and high fractalkine group. We found 25 patients (51\%) were on low fractalkine group and 24 patients (49\%) were on high fractalkine group. Cross tabulation between inflammation group and fractalkine group showed that patients with low inflammation have high fractalkine levels and patients with high inflammation have low fractalkine levels (Figure 1).

Previous research have found that fractalkine levels decreased on 7 days after stroke onset.(13) Based on this finding, we divide subjects into two groups, subjects with stroke onset $<7$ days (A group) and patients with stroke onset 7-30 days (B group). In A group, 9 patients (53\%) have low fractalkine levels, and 8 patients $(47 \%)$ have high fractalkine levels. The same proportion was found in B group, 16 patients $(50 \%)$ have low fractalkine levels and 16 patients $(50 \%)$ have high fractalkine levels. Because of the same percentage in both group, we tried to calculate the mean of fractalkine levels and we found patients on A group have higher mean of fractalkine compared to B group (0.719 $\mathrm{ng} / \mathrm{mL}, 0.585 \mathrm{ng} / \mathrm{mL}$, respectively) (Figure 2), but not significant $(p=0.395)$. Supporting this results, we did cross tabulation between stroke onset group and hsCRP group and we found that patients with stroke onset 7-30 days have high inflammation (Figure 3). In this study, we found that subjects

Table 1. Baseline characteristics of the study population.

\begin{tabular}{lc}
\multicolumn{1}{c}{ Parameters } & $\begin{array}{c}\text { Total Subjects } \\
(\mathbf{n}=49)\end{array}$ \\
\hline Gender $\quad$ Female (n, \%) & $12(24 \%)$ \\
& $37(76 \%)$ \\
Age (year, Mean, range) & $58(38-76)$ \\
DBP (mmHg, Mean, range) & $89(60-159)$ \\
SBP (mmHg, Mean, range) & $144(100-220)$ \\
Hypertension (n, \%) & $23(47 \%)$ \\
Dyslipoproteinemia (n, \%) & $35(71 \%)$ \\
Diabetes (n, \%) & $3(6 \%)$ \\
hsCRP (mg/L, Mean \pm SD) & $10.5 \pm 16.6$ \\
Fractalkine (ng/mL, Mean \pm SD) & $0.635 \pm 0.402$ \\
\hline
\end{tabular}

*DBP: diastolic blood pressure; SBP: systolic blood pressure with stroke onset 7-30 days have higher inflammation than subjects with stroke onset $<7$ days, and subjects with high inflammation have low fractalkine levels. Nevertheless, we didn't found a significant correlation between hsCRP and fractalkine either in A or B group (Table 2).

\section{Discussion}

hsCRP, a newer method for CRP assay, has been proved that higher levels of hsCRP have a strong predictive value in the development of coronary events in the future.(14) It also has been reported that low grade inflammation with raised levels of CRP is an independent risk factor for stroke and TIA.(15) CRP levels also have a tendency to increase with age, reflecting an increased incidence of subclinical pathologic processes.(16) In our study, we found $63.3 \%$ of total subjects with ischemic stroke have high levels of hsCRP $(>3 \mathrm{mg} / \mathrm{L})$ and mean of hsCRP in total subjects are 10.5 $\mathrm{mg} / \mathrm{L}$ (Table 1). Studies of acute ischemic stroke patients have shown varying prevalence and cut off point of hsCRP level. Rajput, et al., had found that among acute ischemic stroke patients from Pakistan, 132 (88\%) had elevated CRP (CRP >10 mg/L).(17) Moreover, in a study by Di Napoli, et al., in Italy, 95 patients (74.2\%) with ischemic stroke had high CRP levels ( $>5 \mathrm{mg} / \mathrm{L}$ ) at admission.(15) Muir, et $a l$., had detected elevated CRP $(>10 \mathrm{mg} / \mathrm{L})$ levels in 96 out of the $228(42.1 \%)$ patients admitted with acute ischemic stroke in the UK.(18) On the other hand, only $22 \%$ of stroke patients had high CRP (>7 mg/L) levels in a study from Netherlands.(19) This variance may be explained partly by the different definitions of high CRP in various studies. In addition, cohort study of stroke patients with median of 10 days after stroke onset demonstrated an association between higher levels of IL-6, CRP and fibrinogen and an increased incidence of recurrent stroke. They found that increase 1 $\mathrm{mg} / \mathrm{L}$ of CRP blood levels had a 1.06-fold increase in the incidence of recurrent vascular events.(20)

The highest plasma fractalkine in all subjects was $2.723 \mathrm{ng} / \mathrm{mL}$. Donohue, et al., reported $514 \mathrm{ng} / \mathrm{mL}$ (range, $367-650 \mathrm{ng} / \mathrm{mL}$ ) as the highest plasma fractalkine by 72 hours onset, but patients with more severe strokes had lower concentrations of plasma fractalkine at day 1 and 7 compared to control subjects.(13) We found patients with stroke onset 7-30 days have mean of fractalkine lower than patients with stroke onset $<7$ days (Figure 2). Other finding on their research, higher fractalkine was associated with a trend toward decreased hsCRP at multiple time points after 


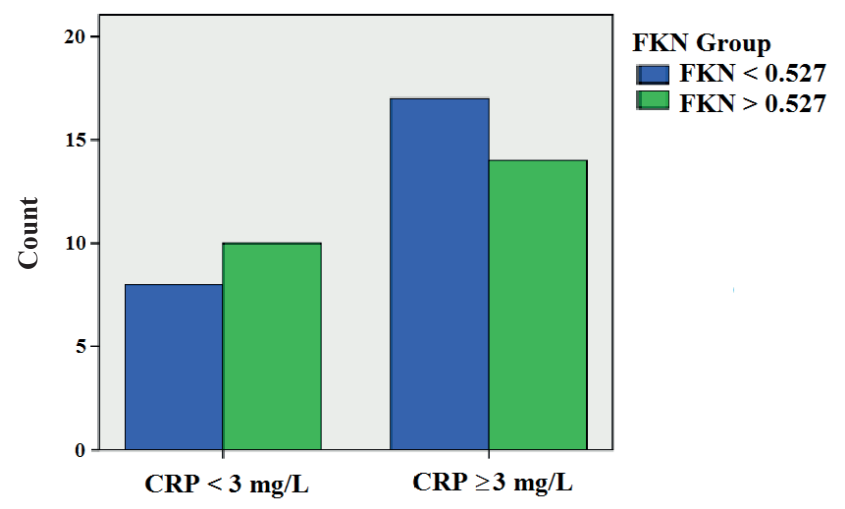

Figure 1. Profile of fractalkine levels in low and high inflammation group. FKN: Fractalkine.

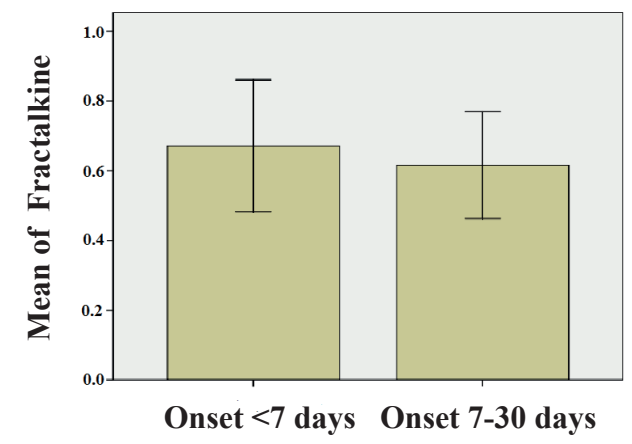

Figure 2. Mean of fractalkine in stroke onset group.

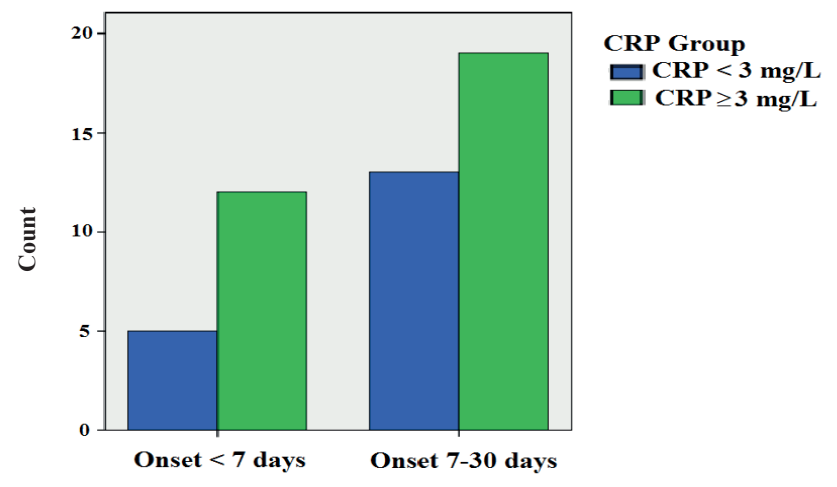

Figure 3. Profile of inflammation in stroke onset group.

stroke and the associations were significant at 180 days after stroke. In our study, we also found that increase of hsCRP patients related with decreased of fractalkine levels on patients with stroke onset 7-30 days (Figure 2 and 3). But we didn't found any correlation between fractalkine and hsCRP in both group of stroke onset (Table 2). This finding was similar with the results of Grosse, et al., that no association of fractalkine and CRP in stroke patients.(21)
The potential contribution of fractalkine to ischemic brain injury has been explored in animal models of stroke. These studies showed that fractalkine expression was upregulated in intact neurons within the penumbra, whereas both CX3CL1 and CX3CR1 expression are upregulated in the infarcted brain, the former in neurons and the latter in microglia.(22) Mice deficient in CX3CL1 have smaller infarct volumes and improved survival after middle cerebral artery occlusion.(23) Our study showed high levels of fractalkine in ischemic stroke patients with stroke onset $<$ 7 days, in line to the theory that fractalkine and its receptor are upregulated in the infarcted brain. However, not many studies have been assesed this chemokine and its role in patients with ischemic stroke.

Fractalkine is constitutively expressed at high levels by neurons, mostly in forebrain structures such as the hippocampus, amygdala, cerebral cortex, globus pallidus, striatum and thalamus, but also in the olfactory bulb, with almost no expression in the cerebellum, at the mRNA and protein levels in adult mouse in situ.(24) In the central nervous system, interaction of fractalkine and its receptor regulates the communication among neurons, glia, and microglia; that is important in the response to injury; and may contribute to neurogenesis. Newly generated cells is significantly reduced in the subgranular zone (SGZ) of 4 month old CX3CR1KO/KO mice compared with CX3CR1KO/+ littermates. Conversely, chronic treatment with fractalkine was found to promote neurogenesis in aged (22 months old) but not young ( 3 months old) or middleaged rats (12 months old), while an antagonist of CX3CR1 produced opposite effects in young, middle-aged and old rats.(25) In a follow-up study, comparing CX3CR1KO/KO and $\mathrm{CX} 3 \mathrm{CR} 1 \mathrm{KO} /+$ mice with wild-type littermates further revealed that adult hippocampal neurogenesis is regulated by fractalkine signaling in a gene-dose dependent manner, with intermediate levels of neurogenesis measured in the heterozygous mice.(24) Although the studied of fractalkine and its receptor in neurogenesis are somewhat contradictory, these findings was support our results that fractalkine levels were upregulated in stroke ischemic patients and decreased 7 days after stroke onset. As fractalkine levels decreased, we found high inflammation on patients with stroke onset 7-30 days. We presumed that the role of fractalkine changes from inflammatory signaling agent in early onset of stroke to promoter of neurogenesis in low concentrations at 7 days after stroke onset, while post-ischemic inflammation will also promote tissue repair in the recovery phase.(26) 
Table 2. Correlation between hsCRP and fractalkine.

\begin{tabular}{ccc}
\hline \multicolumn{1}{c}{ Subjects } & $\boldsymbol{p}$ value* & $\boldsymbol{r}$ \\
\hline Stroke onset $<7$ days & 0.743 & 0.086 \\
Stroke onset $\geq 7$ days & 0.613 & -0.093 \\
\hline
\end{tabular}

*Spearman's rho

There are several limitation of our study. Those are no data of fractalkine levels on healthy subjects as comparation; the wide variation in stroke severity and length time of onset on chronic group, including recurrent stroke; possibility of high concentrations of hsCRP derived from other inflammatory disease or infection that accompanying stroke; and circulating fractalkine obtained from venous blood sample that might be expressed by neurons, endothelial cells, or both.

\section{Conclusion}

In conclusion, we didn't found a significant association between fractalkine and inflammation in stroke ischemic patients. We found a high hsCRP level and low plasma fractalkine profile after 7 days of onset in ischemic stroke patients.

\section{References}

1. Di Carlo A. Human and economic burden of stroke. Age Ageing. 2009; 38: 4-5.

2. Surjawan S, As'ad S, Ranakusuma TAS, Wijaya A. C-reactive protein and matrix metalloproteinase-9 are associated with outcome of ischemic stroke. Indones Biomed J. 2012; 4: 157-63.

3. Iadecola C, Anrather J. The immunology of stroke: from mechanism to translation. Nat Med. 2011; 17: 796-808.

4. Bucova M, Bernadic M, Buckingham T. C-reactive protein, cytokines and inflammation in cardiovascular diseases. Bratisl Lek Listy. 2008; 109: 333-40.

5. Pfutzner A, Forst T. High-sensitivity C-reactive protein as cardiovascular risk marker in patients with diabetes mellitus. Diabetes Technol Ther. 2006; 8(1): 28-36.

6. Pearson TA, Mensah GA, Alexander RW, Anderson JL, Cannon RO 3rd, Criqui M, et al. Markers of inflammation and cardiovascular disease: application to clinical and public health practice: A statement for healthcare professionals from the Centers for Disease Control and Prevention and the American Heart Association. Circulation. 2003; 107: 499-511.

7. Rost NS, Wolf PA, Kase CS, Kelly-Hayes M, Silbershatz H, Massaro JM, et al. Plasma concentration of C-reactive protein and risk of ischemic stroke and transient ischemic attack: the Framingham study. Stroke. 2001; 32: 2575-9.

8. Bazan JF, Bacon KB, Hardiman G, Wang W, Soo K, Rossi D, et al. A new class of membrane-bound chemokine with a CX3C motif. Nature. 1997; 385: 640-4.

9. Fong S, Jones S, Renz ME, Chiu HH, Ryan AM, Presta LG, et al. Mucosal addressin cell adhesion molecule-1 (MADCAM-1). ITS binding motif for alpha 4 beta 7 and role in experimental colitis. Immunol Res. 1997; 16: 299-311.

10. Imai T, Hieshima K, Haskell C, Baba M, Nagira M, Nishimura M, et al. Identification and molecular characterization of fractalkine receptor CX3CR1, which mediates both leukocyte migration and adhesion. Cell. 1997; 91: 521-30.

11. Cardona AE, Pioro EP, Sasse ME, Kostenko V, Cardona SM, Dijkstra IM, et al. Control of microglial neurotoxicity by the fractalkine receptor. Nat Neurosci. 2006; 9: 917-24.

12. Huang $\mathrm{Y}$, Jing J, Zhao XQ, Wang CX, Wang YL, Liu GF, et al. High-sensitivity C-reactive protein is a strong risk factor for death after acute ischemic stroke among Chinese. CNS Neurosci Ther. 2012; 18: 261-6.

13. Donohue MM, Cain K, Zierath D, Shibata D, Tanzi PM, Becker KJ. Higher plasma fractalkine is associated with better 6-month outcome from ischemic stroke. Stroke. 2012; 43: 2300-6.

14. Liuzzo G, Biasucci LM, Gallimore JR, Grillo RL, Rebuzzi AG, Pepys $\mathrm{MB}$, et al. The prognostic value of C-reactive protein and serum amyloid a protein in severe unstable angina. N Engl J Med. 1994; 331: 417-24.

15. Di Napoli M, Papa F, Bocola V. C-reactive protein in ischemic stroke: an independent prognostic factor. Stroke. 2001; 32: 917-24.

16. Koenig W, Sund M, Fröhlich M, Fischer HG, Löwel H, Döring A, et al. C-Reactive protein, a sensitive marker of inflammation, predicts future risk of coronary heart disease in initially healty middleaged men: results from the MONICA (Monitoring Trends and Determinants in Cardiovascular Disease) Augsburg Cohort Study, 1984 to 1992. Circulation. 1999; 99: 237-42.

17. Rajput MR, Lakhair MA, Shaikh MA, Rind MS, Zafarullah, Bano R. C-reactive protein (CRP) and other risk factors in acute ischemic stroke patients. J Liaquat Uni Med Health Sci. 2011; 10: 131-3.

18. Muir KW, Weir CJ, Alwan W, Squire IB, Lees KR. C-reactive protein and outcome after ischemic stroke. Stroke. 1999; 30: 981-5.

19. den Hertog HM, van Rossum JA, van der Worp HB, van Gemert $\mathrm{HM}$, de Jonge R, Koudstaal PJ, et al. C-reactive protein in the very early phase of acute ischemic stroke: association with poor outcome and death. J Neurol. 2009; 256: 2003-8.

20. Whiteley W, Tseng MC, Sandercock P. Blood biomarker in the diagnosis of ischemic stroke: a systematic review. Stroke. 2008; 39: 2902-9.

21. Grosse GM, Tryc AB, Dirks M, Schuppner R, Pflugrad H, Lichtinghagen R, et al. The temporal dynamics of plasma fractalkine levels in ischemic stroke: association with clinical severity and outcome. J Neuroinflam. 2014. 11: 74.

22. Tarozzo G, Campanella M, Ghiani M, Bulfone A, Beltramo M. Expression of fractalkine and its receptor, CX3CR1, in response to ischaemia-reperfusion brain injury in the rat. Eur $\mathrm{J}$ Neurosci. 2002; 15: 1663-8.

23. Soriano SG, Amaravadi LS, Wang YF, Zhou H, Yu GX, Tonra JR, et al. Mice deficient in fractalkine are less susceptible to cerebral ischemia-reperfusion injury. J Neuroimmunol. 2002; 125: 59-65.

24. Bachstetter AD, Morganti JM, Jernberg J, Schlunk A, Mitchell $\mathrm{SH}$, Brewster $\mathrm{KW}$, et al. Fractalkine and $\mathrm{CX} 3 \mathrm{CR} 1$ regulate 
hippocampal neurogenesis in adult and aged rats. Neurobiol Aging. 2011; 32: 2030-44.

25. Rogers JT, Morganti JM, Bachstetter AD, Hudson CE, Peters MM, Grimmig BA, et al. CX3CR1 deficiency leads to impairment of hippocampal cognitive function and synaptic plasticity. J Neurosci. 2011; 31: 16241-50.

26. Shichita T, Sakaguchi R, Suzuki M, Yoshimura A. Post-ischemic inflammation in the brain. Front Immunol. 2012; 3: 132. 